\title{
Comparison of Normative and Diagnosed Dissociation on Attachment to Companion Animals and Stuffed Animals
}

\author{
M. Rose Barlow \\ Boise State University
}

\author{
Lisa DeMarni Cromer \\ University of Tulsa
}

\author{
Hannah Prairie Caron and Jennifer J. Freyd \\ University of Oregon
}

\begin{abstract}
Companion animals can serve as sources of love and attachment during times of stress. Stuffed animals, too, can provide comfort and stability. However, little research has examined companion animal attachment in highly dissociative trauma survivors, and no studies have systematically assessed stuffed animal attachment in dissociative adults. College student samples with either high or low dissociation levels and a sample of women with dissociative identity disorder (DID) completed questionnaires about attachment to companion animals and stuffed animals. The DID group was more attached to companion animals than were either of the student groups. High-dissociating students and DID participants were more attached to stuffed animals than were low-dissociating students. Implications for further research and therapeutic interventions are discussed.
\end{abstract}

Keywords: animal attachment, dissociation, dissociative disorders, stuffed animals, transitional objects

There is a growing interest in animal-assisted therapy in the research literature (e.g., Wesley, Minatreat \& Watson, 2009). Although some of the research relies on small convenience samples, there is reasonable evidence to suggest that attachment to companion animals (but not necessarily animal ownership per se) has positive health impacts. In addition to psychophysiological benefits such as reduced heart rate and lower blood pressure in the presence of companion animals, there are long-term benefits as well, including decreased feelings of loneliness and increased longevity (see Crawford, Worsham, \& Swinehart, 2006, for a review). Positive effects of animal interaction may include better cardiovascular recovery from stress when pets are present, regardless of whether participants report feeling relaxed (Demello, 1999), as well as lower cardiovascular reactivity under stress (Allen, Blascovich, \& Mendes, 2002).

Relationships with companion animals may help to buffer the stress of major aversive life events such as divorce (e.g., Sable, 1995) or even war (Arambašić, Kerestes, Kuterovac-Jagodic, \&

This article was published Online First April 30, 2012.

M. Rose Barlow, Department of Psychology, Boise State University; Lisa DeMarni Cromer, Department of Psychology, University of Tulsa; Hannah Prairie Caron and Jennifer J. Freyd, Department of Psychology, University of Oregon.

We thank Joanna Lahey for valuable comments on a draft of this paper. Funding for part of this research was provided to the first author by a 2005 Dissertation Research Award from the American Psychological Association. Portions of this paper were presented as a poster at the meeting of the American Psychological Association, San Francisco, CA, August 18th, 2007.

Correspondence concerning this article should be addressed to M. Rose Barlow, Department of Psychology, 1910 University Drive, Boise ID 83725-1715. E-mail: rosebarlow@boisestate.edu
Vizek-Vidovic, 2000), and animal-assisted therapy has been used for a variety of psychological conditions (e.g., Lefkowitz, Prout, Bleiberg, Paharia, \& Debiak, 2005; Wesley, Minatrea, \& Watson, 2009). Further, companion animals have been shown in numerous studies to serve as objects of love, friendship, and attachment (see Enders-Slegers, 2000, for a review). In fact, attachment relationships between people and companion animals may be more secure than relationships with romantic partners (Beck \& Madresh, 2008). There appears to be an association between pet attachment and decreased feelings of loneliness, isolation, and increases in mood (possibly as a result of increases in physical activity, such as dog walking) (Krause-Parello, 2008; le Roux \& Kemp, 2009).

In addition, companion animals may support socioemotional development in children (Beck \& Katcher, 2003; Budge, Spicer, Jones, \& St. George, 1998; Demello, 1999). In early childhood, preschoolers articulate a great deal of love and affection between themselves and their pets (Triebenbacher, 1998). The preadolescent human-animal bond is characterized as a "friendship" that is best described as mutual love and unconditional acceptance (Davis \& Juhasz, 1995). Unconditional love and affection from animals were also shown to be the major benefits of human-animal relationships in a study of adult cat owners (Zasloff \& Kidd, 1994). Given the benefits of companion animals in normative development, it is surprising that, to date, only a handful of studies have examined attachment to companion animals in relation to development in an atypical context, such as when children experience abuse or neglect. It is possible that the human-animal bond could serve as an attachment relationship and a means of support to buffer the negative effects of adverse life experiences.

Raupp (1999) found that parental attitudes toward animals, whether negative or positive, are often passed down to their children. Participants who formed weaker attachments to companion animals were more likely to mistreat them. Abusing animals is 
often an early warning of violence toward humans. For example, in a study conducted in a maximum-security prison, there was a statistically significant relationship between maltreatment of animals in childhood and violent crimes in adulthood, and this effect was especially pronounced when the animal was a pet (MerzPerez, Heide, \& Silverman, 2001).

An emerging literature examines dissociation and animal attachment. The Diagnostic and Statistical Manual of Mental Disorders$I V-T R$ describes dissociation as a separation between processes that are normally integrated, such as events, emotions, and memories (American Psychiatric Association, 2000). Survivors of severe trauma, especially interpersonal trauma such as child abuse, may experience high levels of dissociation, that is, a prominent lack of connection between such areas of function as perception, memory, and sense of selfhood (e.g., Chu \& Dill, 1990; Draijer \& Langeland, 1999; Kisiel \& Lyons, 2001). Nonetheless, dissociation is not always pathological. In fact, it has been argued that dissociation may be best understood as a continuum from more common, normal manifestations to less common and more pathological symptoms (e.g., Dalenberg \& Paulson, 2009).

In examining dissociation in normal populations, some authors have found that dissociation is related to attachment to companion animals. Brown and Katcher (1997) found that, in a sample of college students, dissociation was positively related to companion animal attachment. The association between animal attachment and dissociation was replicated in another student sample (Brown \& Katcher, 2001). Within nonclinical samples, there are often individuals who score high on measures of dissociation. In nonclinical college student samples, researchers found that the frequency of clinical levels of dissociation was significantly higher in participants with the highest levels of companion animal attachment than in participants with lower levels of attachment (Brown $\&$ Katcher, 1997, 2001). The authors speculated that early attachment problems could lead people to become highly attached to companion animals as a substitute for human relationships. They also suggested that the relationships people have with animals may include dissociative qualities, such as imaginative involvement and fantasy. Moreover, the physiological impacts of interaction with animals may mimic dissociative states (Brown \& Katcher, 2001). Unfortunately, this correlational research does not eliminate the possibility of a third variable (e.g., imaginative involvement) that could lead individuals to be more highly attached to companion animals and also to have higher scores on measures of dissociation.

Subsequent work attempted to further explore the relationship of companion animal attachment to dissociation in nonclinical samples (Cromer \& Freyd, 2004). In two studies, companion animal attachment was positively correlated with overall level of dissociation, as well as with three dissociation subscales: imaginative involvement, depersonalization, and dissociative activities. The correlations between aspects of dissociation and animal attachment were statistically significant for the first two subscales, supporting Brown and Katcher's theories about dissociative qualities of these human-animal relationships (Cromer \& Freyd, 2004). Although this research supports the relationship between a global concept of dissociation and attachment to animals, it does not determine causality.

The present study seeks to further our understanding of the relationship between animal attachment and trauma history, in particular as trauma relates to dissociative tendencies. Some pre- vious research on animal attachment and dissociation has used small convenience samples that limit generalizability. For example, researchers have recruited volunteer samples of pet owners (Davis \& Juhasz, 1995; Holcomb, Williams, \& Richards, 1985; Zasloff \& Kidd, 1994) or veterinary students (Brown, 2002; Brown \& Katcher, 2001). The current study extends previous research by examining the relation between dissociation and animal attachment in two different populations. One population, college students, has often been included in previous research, but in the current study the sample was recruited based on dissociation scores instead of pet ownership or knowledge of the study. The other sample in the current study consisted of women with clinically confirmed diagnoses of dissociative identity disorder (DID) formerly called multiple personalities). Individuals with DID have not been included in research on animal attachment, despite the importance of studying animal attachment in trauma survivors. As people with DID have usually experienced high levels of trauma, examining this group may provide a window into severe dissociative responses and companion animal attachment.

Living with a companion animal is not always an option. Life circumstances or transitions, such as the transition out of a family home and into a college dormitory, can preclude owning live animals. In these cases, stuffed animals such as teddy bears may provide an analogous form of comfort that could otherwise be derived from companion animals. Stuffed animals may provide comfort and stability for survivors of interpersonal trauma; however, no studies have systematically assessed stuffed animal attachment in dissociative adults. Stuffed animals may play an important role as transitional objects. Winnicott (1953) theorized that attachment objects help infants reduce anxiety and feel secure and in control as they develop separateness and independence from their mothers. Transitional objects in childhood typically provide tactile comfort during periods of stress or transition to sleep.

Stuffed animals are common transitional objects for children and adolescents (Cromer \& Freyd, 2004). A previous study found that, despite relatively high rates of stuffed animal ownership, stuffed animals may serve as transitional objects only for some individuals. Cromer and Freyd found that participants who selfreported high levels of attachment to their stuffed animals had higher rates of dissociation than did individuals who were not attached to their stuffed animals (Cromer \& Freyd, 2004). A possible explanation for these attachment differences to stuffed animals could relate to differences in coping, where stuffed animals serve as transitional objects (Triebenbacher, 1998). We were interested in examining whether there is a correspondence between stuffed animal attachment and attachment to companion animals.

This study also extends previous research by examining selfreported dissociation level in relation to both companion animal attachment and stuffed animal attachment. A more thorough understanding of the relations among dissociation, stuffed animal attachment, and animal attachment could be beneficial for therapy and recovery from trauma. Previous research has found that college student participants who were highly dissociative were more likely than low dissociators to view stuffed animals as friends and to take their stuffed animals to bed with them (Cromer \& Freyd, 2004). This finding suggests that comfort objects such as stuffed animals could function during transitions throughout the life span, such as beginning college. We were particularly interested in how attachment to objects and animals related to dissociation, as this 
relationship could further illuminate unique characteristics of individuals who are more dissociative than others.

Based on the previous literature regarding associations among dissociation and animal attachment, this study had two hypotheses. The first hypothesis was that dissociation would be positively correlated with companion animal attachment, replicating the findings of previous research and extending it into a clinical sample. The second hypothesis was that participants with higher dissociation would be more attached to stuffed animals than would participants with low levels of dissociation.

\section{Method}

\section{Participants}

Student participants. Seventy-two college student participants from the University of Oregon human subject pool were recruited via phone and email on the basis of their scores on a dissociative measure during a general online prescreening. High and low dissociators as measured by the DES (described below) were recruited for the study. A cutoff score of $\leq 10$ on the DES was used for low dissociators, and a cutoff score of $\geq 20$ was used for high dissociators.

The low-dissociation group ( $n=37 ; 26$ female) had a mean age of $19.32(S D=1.56)$, and the high-dissociation group $(n=35 ; 25$ female) had a mean age of $19.26(S D=1.27)$. The dissociation scores as measured by the DES were, for the low dissociators, $M=$ $8.69(S D=3.12)$, and for the high dissociators, $M=33.25(S D=$ 14.14).

DID participants. Recruitment for this group of participants took place as part of a larger research study on cognitive processing in DID. There were two waves of recruitment for these participants. Inpatient recruitment took place at a psychiatric hospital in the Boston area, and resulted in one participant. Then, outpatient recruitment took place in the Eugene, Portland (OR), and Seattle areas. A letter explaining the overall project was sent to treatment providers that specialized in dissociation or trauma. Information was also sent out over an electronic mailing list and via a website. All participants had been diagnosed by their individual treating clinicians, using DSM criteria.

The resulting DID group consisted of 11 women who had been diagnosed with DID. One was an inpatient at a psychiatric hospital in Massachusetts, and the rest were community members in the Pacific Northwest. One participant did not answer demographic questions. The mean age was 35.35 years $(S D=12.57$, range $=$ 23-62). The DID group had one member with a high school education or GED, four people with some college or technical school, two people who had completed college or technical school, two people with some graduate school, and one person who had completed graduate school. This group had been in therapy for an average of 12.05 years $(S D=6.73$, range $=2-20$ years $)$. The DID group had a mean DES score of $56.16(S D=21.88)$, which is well above the normal range.

\section{Materials}

Pet Attachment and Life Impact Scale (PALS; Cromer \& Freyd, 2004). The PALS is a 30 -item, five-point (0 to 4) Likert-type scale measuring relational and emotional attachment to pets as well as negative impacts of pets on people's lives (reverse scored items). This measure was empirically developed over a period of two years across several studies (Cromer \& Freyd, 2004). The PALS incorporates eight items from the Pet Attachment Questionnaire (Stallones, Johnson, Garrity, \& Marx, 1990) in addition to 22 items that were generated from responses to the open-ended question, "how have pets impacted your life?"

Stuffed Animal Attachment Questionnaire (SAQ; Cromer \& Freyd, 2004). The SAQ (Cromer \& Freyd, 2004) is an empirically developed 20 -item questionnaire that asks about attachment to stuffed animals. Responses are provided on a four-point (1-4) Likert scale. Examples of items are: "My stuffed animals have personalities all their own," "Stuffed animals can give you love," and "I somehow feel safer with a stuffed animal."

Dissociative Experiences Scale (DES; Bernstein \& Putnam, 1986). The DES is a 28 -item self-report measure that can be used to assess different types of dissociative experiences, ranging from "spacing out" to amnesia for important autobiographical events. It has been used extensively with a wide range of populations in countries around the world and has been found to have strong reliability and validity (see Briere, 1997; Carlson \& Putnam, 1993 for reviews). This scale was not designed for diagnostic use, and the general population of adults usually scores in a narrow range below 10 (Carlson \& Putnam, 1993). van IJzendoorn and Schuengel (1996) reported that the mean alpha reliability of the DES in their meta-analysis was .93.

\section{Procedure}

Student participants completed the surveys in the laboratory, with the experimenter being blind to whether participants were high or low dissociators. Participants with DID completed the questionnaires at the end of the second session in a larger study of DID. All participants gave ongoing informed consent. After debriefing, student participants received credit toward a class requirement and DID participants were paid for their time. This research was approved by the University of Oregon Institutional Review Board and the Institutional Review Board of McLean Hospital.

\section{Results}

Overall, attachment to pets and attachment to stuffed animals showed a medium and statistically significant correlation $(r=.35$, $p<.02)$. The omnibus multivariate test was significant, Wilk's $\lambda=.76, F(4,156)=5.64, p<.001$, partial eta squared $=.13$. The test of between-subjects effects was also significant for both the pet attachment measure, $F(2,79)=5.22, p<.01$, partial eta squared $=.12$, and the stuffed animal attachment measure, $F(2$, $79)=8.13, p<.001$, partial eta squared $=.17$. See Figures 1 and 2 for mean scores of companion animal and stuffed animal attachment. Tukey's HSD post hoc tests revealed that the DID group was higher than low dissociators ( $p<.01, d=1.11$ ) and high dissociators $(p<.05, d=.92)$ on pet attachment, whereas the two groups of college students were not statistically different from each other, though the trend was in the same direction.

On stuffed animal attachment, Tukey's HSD found that the low dissociators were less attached to stuffed animals than were the high dissociators $(p<.01, d=.87)$ and the DID group $(p<.05$, 


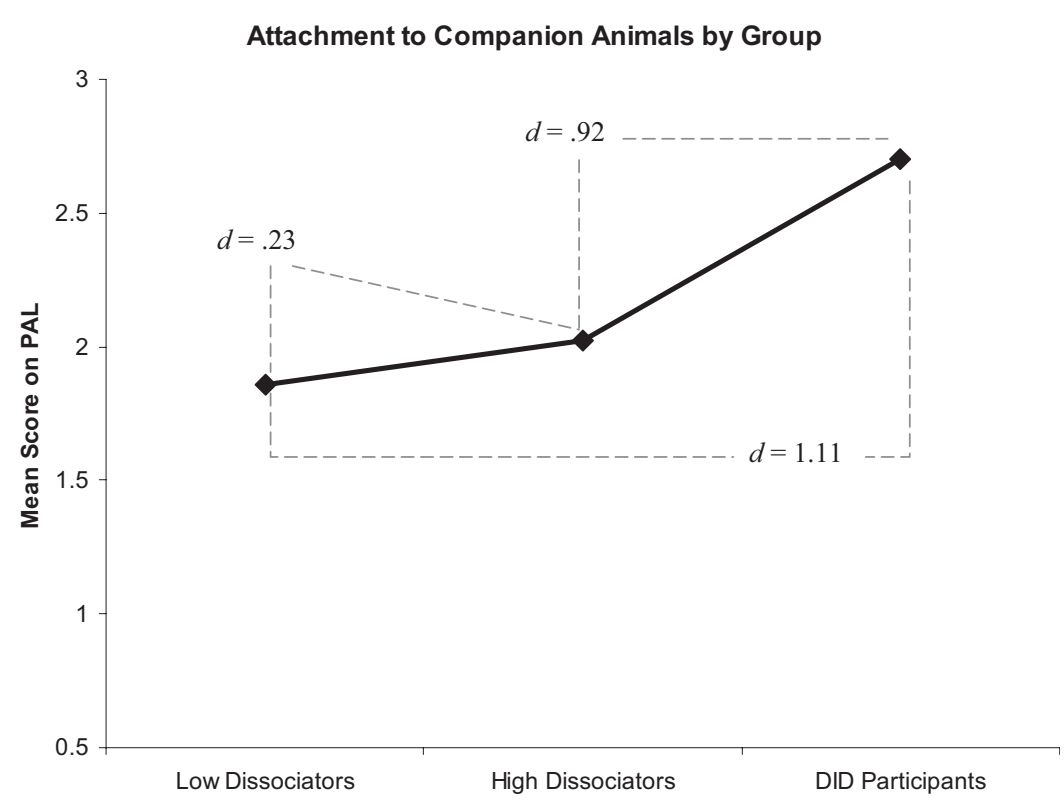

Figure 1. Attachment to companion animals by group.

$d=1.05$ ). The high dissociators and the DID group did not significantly differ from each other, though the trend was in the same direction. Post hoc regression analyses revealed that this linear relationship across groups was driven by differences in the Absorption-Imaginative Involvement Subscale of the DES (see Figure 2).

\section{Discussion}

Our first hypothesis was partially supported. The DID group was more attached to companion animals than were either of the student groups, with very large effect sizes. The difference between low and high dissociators in the student group was not statistically significant, although the trend was in the predicted direction. The second hypothesis was supported: high-dissociating students and DID participants were more attached to stuffed animals than were the low-dissociating students, again with large effect sizes.

This study is the first to assess companion animal attachment systematically in a DID sample. It is also the first to assess attachment to stuffed animals in a DID sample and to compare their level of attachment to that of a nonclinical sample. This study supports earlier work by Cromer and Freyd (2004), which found that high dissociators in a college sample were more likely than were low dissociators to view stuffed animals as friends and as a

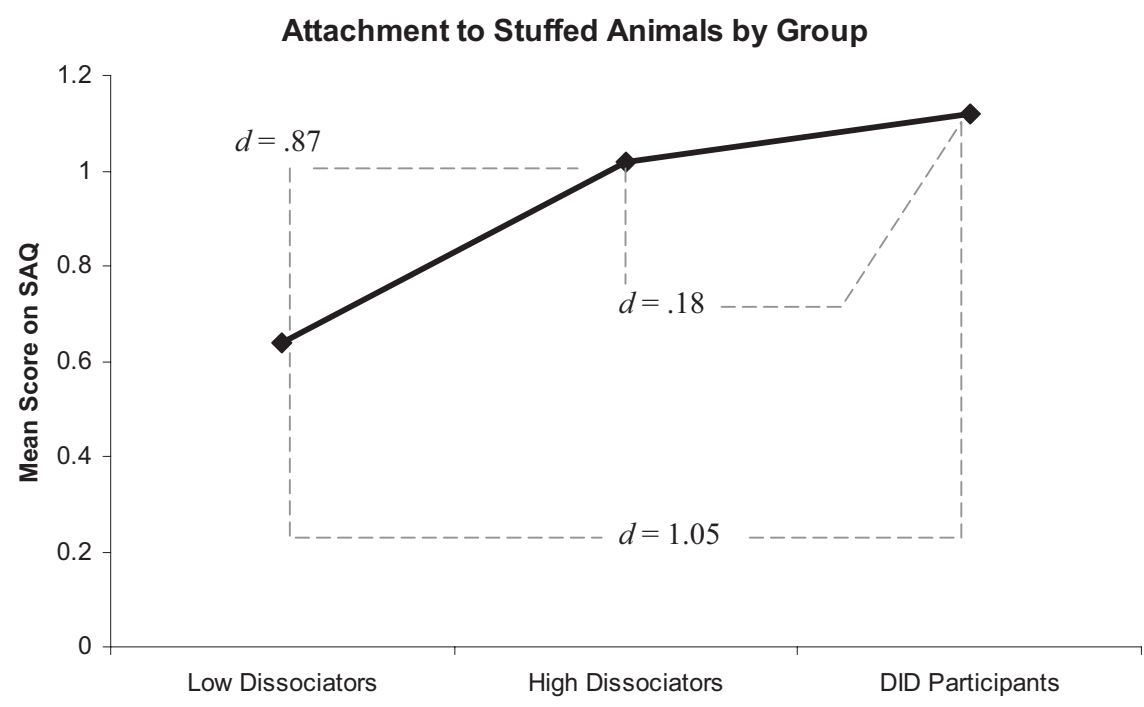

Figure 2. Attachment to stuffed animals by group. 
comfort; in other words, having an emotional relationship with stuffed animals was associated with high levels of dissociation. The current study also supports a theoretical viewpoint of stuffed animal attachment as a coping mechanism to deal with both trauma and with the dysregulated affect that is part of severe dissociation.

One limitation of this study is that it does not rule out a link between attachment and nonspecific psychopathology. Given that DID has a very high incidence of comorbidity with mood, anxiety, and other disorders (e.g., Sar, Koyuncu, et al., 2007; Sar, Akyuz, \& Dogan, 2007), it would be helpful to examine whether dissociation itself was the driving force behind our effects, or whether these other factors could account for the attachment findings. People with DID may also show a high rate of Schneiderian first-rank symptoms (e.g., Sar, Koyuncu, et al., 2007; Sar, Yargic, $\&$ Tutkun, 1996), and these atypical ways of viewing the world could also play a role in unusual attachment. Future research should endeavor to untangle these complex relationships.

Brown and Katcher (1997) proposed that becoming highly attached to companion animals can result from problems in early attachment relationships, which fits with a view of pets and stuffed animals as transitional objects (Winnicott, 1953). One study found that young children in single-parent families were more attached to their dogs than were children with two parents in the home (Bodsworth \& Coleman, 2001). Dissociation may begin with an early disorganized attachment, even if no overt abuse is occurring (Hesse \& Main, 2000; Liotti, 1999). Disorganized attachment therefore increases vulnerability to dissociative disorders but is not in itself sufficient to cause a disorder without additional trauma (Hesse \& Main, 2000).

Support for this model of dissociation as a response to attachment problems was also found in a study that showed that a familial pattern of disorganized attachment may predispose children to dissociate in response to later trauma (Pasquini, Liotti, Mazzotti, Fassone, Picardi, et al., 2002). In fact, recent results from a longitudinal study showed that children of mothers who were unresponsive during infancy had higher rates of dissociation in young adulthood nearly two decades later (Dutra, Bureau, Holmes, Lyubchik, \& Lyons-Ruth, 2009). Infancy variables of maternal flat affect, maternal lack of positive affect, and disrupted communication between mother and infant collectively accounted for fully half the variance in young adult dissociation, even after controlling for other familial risk factors (Dutra et al., 2009). Dutra and colleagues (2009) suggested that dissociation arises dynamically from the context of disturbed interpersonal interaction in a vital attachment relationship. Supporting this theory too, Cromer and Freyd (2004) found that while stuffed animal attachment was related to dissociation, mere ownership of stuffed animals was not. While many people own stuffed animals, having relational interactions with them is less common. Again, attachment dynamics seem to play a vital role in forming dissociative responses.

People with DID may sometimes project identity states into stuffed animals, and sometimes they carry stuffed animals to represent parts of themselves, or for protection. ${ }^{1}$ In light of this clinical observation and the attachment theories mentioned above, it is important that future research address the potential usefulness of stuffed animals as an aid to daily functioning and as an adjunct to therapy. There are anecdotal reports of the usefulness of stuffed animals in therapy for dissociative disorders (Arnold, 1995), and this area should be examined in future studies. Indeed, a recent study suggests that traumatized children may benefit from stuffed animal attachment (Sadeh, Hen-Gal, \& Tikotzky, 2008). Children between two and seven years old who had been displaced to a camp during a war showed faster reduction of stress responses when they were given a stuffed animal and encouraged to care for it, compared with children who did not receive the stuffed animal. The benefits included lowered anxiety, less nervousness and aggression, and less crying (Sadeh et al., 2008). Although more research is needed to replicate this result, taken with the present study it suggests that helping children establish attachment to a transitional object during a time of attachment disruptions could be a viable intervention or a supplement to other interventions.

This line of research may open the door for replicating clinical pet-intervention studies with stuffed animal interventions. Understanding which people are more likely to become attached to stuffed animals or to live therapy animals may help determine which clients are most likely to benefit from having a live or stuffed animal as part of their therapy. Those who are most attached to animals, or who have the potential to become attached, could participate in clinical interventions using therapy dogs or other animals, their own animals, or even stuffed animals. As previous research has shown, interactions with animals can have many benefits. These benefits may be differentially powerful or helpful for people who have endured the social distancing and sense of fragmentation caused by surviving trauma and high levels of dissociation. Animals, live or stuffed, can aid therapy for both children and adults by providing a way to experience and express emotions, a feeling of unconditional support, and grounding. Future research should examine in detail the potential uses of stuffed animals in therapeutically regulating dissociative processes. Examining physiological reactions to stress and the emergence or strength of dissociative states in the presence of a stuffed animal would be a valuable beginning to understanding this area, and future research will examine this topic.

${ }^{1}$ We thank Dr. Christine Courtois for bringing this point to our attention.

\section{References}

Allen, K., Blascovich, J., \& Mendes, W. B. (2002). Cardiovascular reactivity and the presence of pets, friends, and spouses: The truth about cats and dogs. Psychosomatic Medicine, 64, 727-739. doi:10.1097/ 01.PSY.0000024236.11538.41

American Psychiatric Association (2000). Diagnostic and statistical manual of mental disorders (4th edition, text revision). Washington, DC: Author.

Arambašić, L., Kerestes, G., Kuterovac-Jagodic, G., \& Vizek-Vidovic, V. (2000). The role of pet ownership as a possible buffer variable in traumatic experiences. Studia Psychologica, 42, 135-146.

Arnold, J. C. (1995). Therapy dogs and the dissociative patient: Preliminary observations. Dissociation: Progress in the Dissociative Disorders, $8,247-252$.

Beck, A. M., \& Katcher, A. H. (2003). Future directions in human-animal bond research. American Behavioral Scientist, 47, 79-93. doi:10.1177/ 0002764203255214

Beck, L., \& Madresh, E. A. (2008). Romantic partners and four-legged friends: An extension of attachment theory to relationships with pets. Anthrozoös, 21, 43-56. doi:10.2752/089279308X274056

Bernstein, E. M., \& Putnam, F. W. (1986). Development, reliability, and 
validity of a dissociation scale. Journal of Nervous and Mental Disease, 174, 727-735. doi:10.1097/00005053-198612000-00004

Bodsworth, W., \& Coleman, G. J. (2001). Child-companion animal attachment bonds in single and two-parent families. Anthrozoös, 14, 216-223. doi:10.2752/089279301786999391

Briere, J. (1997). Psychological assessment of adult posttraumatic states. Washington, DC: American Psychological Association. doi:10.1037/ 10267-000

Brown, S. E., \& Katcher, A. H. (1997). The contribution of attachment to pets and attachment to nature to dissociation and absorption. Dissociation: Progress in the Dissociative Disorders, 10, 125-129.

Brown, S. E., \& Katcher, A. H. (2001). Pet attachment and dissociation. Society \& Animals: Journal of Human-Animal Studies, 9, 25-41. doi: $10.1163 / 156853001300108973$

Brown, S. E. (2002). Ethnic variations in pet attachment among students at an American school of veterinary medicine. Society \& Animals: Journal of Human-Animal Studies, 10, 249-266. doi:10.1163/156853002320770065

Budge, R. C., Spicer, J., Jones, B., \& St. George, R. (1998). Health correlates of compatibility and attachment in human-companion animal relationships. Society \& Animals: Journal of Human-Animal Studies, 6, $219-234$

Carlson, E. B., \& Putnam, F. W. (1993). An update on the Dissociative Experiences Scale. Dissociation: Progress in the Dissociative Disorders, 6, 16-27.

Chu, J. A., \& Dill, D. L. (1990). Dissociative symptoms in relation to childhood physical and sexual abuse. The American Journal of Psychiatry, 147, 887-892.

Crawford, E. K., Worsham, N. L., \& Swinehart, E. R. (2006). Benefits derived from companion animals, and the use of the term "attachment." Anthrozoös, 19, 98-112. doi:10.2752/089279306785593757

Cromer, L. D., \& Freyd, J. J. (2004, February). Stuffed animals, pets, and dissociation. Poster presented at the American Association for the Advancement of Science annual conference, Seattle, WA.

Dalenberg, C. J., \& Paulson, K. (2009). The case for the study of "normal" dissociation processes. In P. F. Dell \& J. A. O'Neil (Eds.), Dissociation and the dissociative disorders: DSM-V and beyond (pp. 145-154). New York, NY: Routledge.

Davis, J. H., \& Juhasz, A. M. (1995). The preadolescent/pet friendship bond. Anthrozoös, 8, 78-82. doi:10.2752/089279395787156437

Demello, L. R. (1999). The effect of the presence of a companion animal on physiological changes following the termination of cognitive stressors. Psychology \& Health, 14, 859-868. doi:10.1080/08870449908407352

Draijer, N., \& Langeland, W. (1999). Childhood trauma and perceived parental dysfunction in the etiology of dissociative symptoms in psychiatric inpatients. The American Journal of Psychiatry, 156, 379-385.

Dutra, L., Bureau, J.-F., Holmes, B., Lyubchik, A., \& Lyons-Ruth, K. (2009). Quality of early care and childhood trauma: A prospective study of developmental pathways to dissociation. Journal of Nervous and Mental Disease, 197, 383-390. doi:10.1097/NMD.0b013e3181a653b7

Enders-Slegers, M. (2000). The meaning of companion animals: Qualitative analysis of the life histories of elderly cat and dog owners. In A. L. Podberscek \& E. S. Paul (Eds.), Companion animals and us: Exploring the relationship between people and pets (pp. 237-256). London, UK: Cambridge University Press.

Hesse, E., \& Main, M. (2000). Disorganized infant, child, and adult attachment: Collapse in behavioral and attentional strategies. Journal of the American Psychoanalytic Association, 48, 1097-1127. doi:10.1177/ 00030651000480041101

Holcomb, R., Williams, R. C., \& Richards, P. S. (1985). The elements of attachment: Relationship maintenance and intimacy. Journal of the Delta Society, 2, 28-34

Kisiel, C. L., \& Lyons, J. S. (2001). Dissociation as a mediator of psychopa- thology among sexually abused children and adolescents. The American Journal of Psychiatry, 158, 1034-1039. doi:10.1176/appi.ajp.158.7.1034

Krause-Parello, C. A. (2008). The mediating effect of pet attachment support between loneliness and general health in older females living in the community. Journal of Community Health Nursing, 25, 1-14.

Lefkowitz, C., Prout, M., Bleiberg, J., Paharia, I., \& Debiak, D. (2005). Animal-assisted prolonged exposure: A treatment for survivors of sexual assault suffering posttraumatic stress disorder. Society \& Animals: Journal of Human-Animal Studies, 13, 275-296. doi:10.1163/156853005774653654

le Roux, M. C., \& Kemp, R. E. (2009). Effect of a companion dog on depression and anxiety levels of elderly residents in a long-term care facility. Psychogeriatrics, 9, 23-26. doi:10.1111/j.1479-8301.2009.00268.x

Liotti, G. (1999). Understanding the dissociative processes: The contribution of attachment theory. Psychoanalytic Inquiry, 19, 757-783. doi: 10.1080/07351699909534275

Merz-Perez, L., Heide, K. M., \& Silverman, I. J. (2001). Childhood cruelty to animals and subsequent violence against humans. International Journal of Offender Therapy and Comparative Criminology, 45, 556-573. doi:10.1177/0306624X01455003

Pasquini, P., Liotti, G., Mazzotti, E., Fassone, G., Picardi, A., \& The Italian Group for the Study of Dissociation. (2002). Risk factors in the early family life of patients suffering from dissociative disorders. Acta Psychiatrica Scandinavica, 105, 110-116. doi:10.1034/j.1600-0447.2002 $.01062 . \mathrm{x}$

Raupp, C. D. (1999). Treasuring, trashing, or terrorizing: Adult outcomes of childhood socialization with animals. Society \& Animals: Journal of Human-Animal Studies, 7, 141-159. doi:10.1163/156853099X00040

Sable, P. (1995). Pets, attachment, and well-being across the life cycle. Social Work, 40, 334-341.

Sadeh, A., Hen-Gal, S., \& Tikotzky, L. (2008). Young children's reactions to war-related stress: A survey and assessment of an innovative intervention. Pediatrics, 121, 46-53. doi:10.1542/peds.2007-1348

Sar, V., Akyuz, G., \& Dogan, O. (2007). Prevalence of dissociative disorders among women in the general population. Psychiatry Research, 149, 169-176. doi:10.1016/j.psychres.2006.01.005

Sar, V., Koyuncu, A., Ozturk, E., Yargic, L. I., Kundakci, T., Yazici, A., ... Aksuit, D. (2007). Dissociative disorders in the psychiatric emergency ward. General Hospital Psychiatry, 29, 45-50. doi:10.1016/ j.genhosppsych.2006.10.009

Sar, V., Yargic, I., \& Tutkun, H. (1996). Structured interview data on 35 cases of dissociative identity disorder in Turkey. The American Journal of Psychiatry, 153, 1329-1333.

Stallones, L., Johnson, T. P., Garrity, T. F., \& Marx, M. B. (1990). Quality of attachment to companion animals among U.S. adults 21 to 64 years of age. Anthrozoös, 3, 171-176. doi:10.2752/089279390787057595

Triebenbacher, S. L. (1998). Pets as transitional objects: Their role in children's emotional development. Psychological Reports, 82, 191-200.

van IJzendoorn, M. H., \& Schuengel, C. (1996). The measurement of dissociation in normal and clinical populations: Meta-analytic validation of the Dissociative Experiences Scale (DES). Clinical Psychology Review, 16, 365-382. doi:10.1016/0272-7358(96)00006-2

Wesley, M. C., Minatrea, N. B., \& Watson, J. C. (2009). Animal-assisted therapy in the treatment of substance dependence. Anthrozoös, 22, 137-148. doi:10.2752/175303709X434167

Winnicott, D. W. (1953). Transitional objects and transitional phenomena: A study of the first not-me possession. The International Journal of Psychoanalysis, 34, 89-97.

Zasloff, R. L., \& Kidd, A. H. (1994). Attachment to feline companions. Psychological Reports, 74, 747-752. doi:10.2466/pr0.1994.74.3.747

Received January 27, 2010 Accepted April 7, 2010 